\title{
Extra-functional contract support in components
}

\author{
Olivier Defour, Jean-Marc Jézéquel, Noël Plouzeau \\ INRIA-Rennes, Campus universitaire de Beaulieu, Avenue du général Leclerc \\ 35042 Rennes Cedex, France \\ \{olivier.defour, jean-marc.jezequel, noel.plouzeau\}@irisa.fr \\ http://www.irisa.fr/triskell
}

\begin{abstract}
According to Szyperski, "a software component is a unit of composition with contractually specified interfaces and explicit context dependencies only". But it is well known that these contractually specified interfaces should go well beyond mere syntactic aspects: they should also involve functional, synchronization and Quality of Service (QoS) aspects. In large, mission-critical component based systems, it is also particularly important to be able to explicitly relate the QoS contracts attached to provided interfaces with the QoS contracts obtained from required interfaces. In this paper we propose a language called QoSCL (defined as an add-on to the UML2.0 component model) to let the designer explicitly describe and manipulate these higher level contracts and their dependencies. We show how the very same QoSCL contracts can then be exploited for validation of individual components and also validation of a component assembly, including getting end-to-end QoS information inferred from individual component contracts, by automatic translation to a Constraint Logic Programming language. We illustrate our approach with the example of a GPS (Global Positioning System) software component, from its functional and contractual specifications to its implementation in a .Net framework.
\end{abstract}

\section{Introduction}

In Szyperski's vision [1], "a software component is a unit of composition with contractually specified interfaces and explicit context dependencies only. A software component can be deployed independently and is subject to composition by thirdparty". In this vision, any composite application is viewed as a particular configuration of components, selected at build-time and (re-)configured at run-time.

This point of view is now widely adopted in components middleware technologies such as Corba Component Model (CCM) [2], Entreprise JavaBean (EJB) [3] or Microsoft .Net/Com [4]. In these various middleware, a software component is a binary executable code deployed in an environment which manages it (EJBContainer for EJB or component home for CCM). A software component only exhibits its provided or 
required interfaces, hence defining basic contracts between components allowing one to properly wire them. But it is well known that these contractually specified interfaces should go well beyond mere syntactic aspects: they should also involve functional, synchronization [5] and Quality of Service (QoS) aspects [6]. In large, mission-critical component based systems, it is also particularly important to be able to explicitly relate the QoS contracts attached to provided interfaces with the QoS contracts obtained from required interfaces.

The aim of this article is to present a QoS contract model (called QoSCL for QoS Constraint Language), allowing such QoS contracts and their dependencies to be specified at design-time in a UML2.0 [7] modeling environment. We then show how the very same QoSCL contracts can be exploited not only for validation of individual components but also validation of a component assembly, including getting end-toend QoS information inferred from individual component contracts, by automatic translation to a Constraint Logic Programming [8].

The rest of the paper is organized as follows. Using the example of a GPS (Global Positioning System) software component, Section 2 introduces the interest of modelling components with their contracts and their dependencies, and describes the QoS Constraint Language (QoSCL). Section 3 discusses the problem of validating a component assembly, including getting end-to-end QoS information inferred from individual component contracts by automatic translation to a Constraint Logic Programming (CLP). This is applied to the GPS system example, and experimental results are presented. Finally, Section 4 presents related works.

\section{QoS specification}

\subsection{The common QoS features}

Before to introduce the metamodel of any specification language, it is important to understand the semantic of the concepts that are handled. What are the concepts that we want to specify, their semantic, their features, properties and relationships?

The quality of a service is defined as a set of extra-functional properties. Consequently, an extra-functional property is an intrinsic qualitative dimension of a service. An extra-functional property especially is a valuable quantity. This aspect implies that:an extra-functional property is ever associated to means of measurement;

2. as any valuable quantity, an extra-functional property can be constrained;

3. the effective quality of a service is the value of its extra-functional properties, compared to a reference totally ordered scale of values: the quality levels.

A quality level is a set of values, denumerable or not, and usually bounded. For instance, the getLocation() service provided by the GPS component, shown in Fig. 1 below, can be qualified by two extra-functional properties: the precision eps of the estimated position, and the response time $\theta_{\mathbf{C}}$. The precision is defined in the finite set 
\{low; normal; high , with the total following order: low $<$ normal $<$ high. The response time is a positive real value, dedicated to be less than a specified time out value.

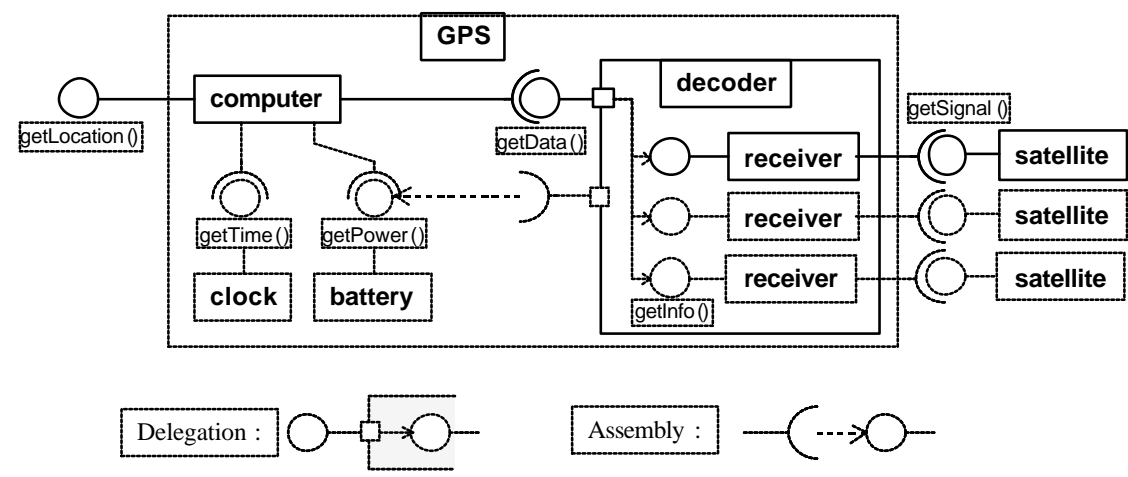

Fig. 1. Component-based model of a GPS device

Any artefact that realizes the GPS component model must implement not only the getLocation service, but also a means of measurement for its two intrinsic extrafunctional properties eps and $\theta_{\mathbf{C}}$. Consequently, it is useful to consider an extrafunctional property as the specification of its means of measurement: the semantic of what is measured is defined at the component model level refined with QoS specifications.

A QoS specification languages, such as QML [9] for exa mple, is dedicated no only to specify extra-functional properties, but also a set of constraints. Usually, these constraints are checked at run time, in order to renegotiate contracts. At design time, the constraints are used to specify either qualitative requirements for required services, or provided quality levels for provided services. At the assembly level between two components, we must check that the quality level of the provided service is compliant with the qualitative requirements.

For instance, the GPS component is connected to a set of satellite components that provide the required getSignal services. Let $\theta_{\mathrm{S}}$ be the response time of the getSignal service provided by the satellite components. A signal emitted by a satellite has a duration of $15 \mathrm{~ms}$ and, so, the GPS receives a complete signal in a time greater than 15 $\mathrm{ms}$ and less than $30 \mathrm{~ms}$. That is a quality level of the provided getSignal service. But the GPS can also requires that the response time to complete the signal must be less than 20 ms: in this case, the constraint on the response time of the getSignal service is not compliant with the provided QoS level.

An other main feature of the QoS is the extra-functional dependency. A service provided by a component usually depends on the a subset of services that it requires. A component-based model refined enough, as the Fig. 1, shown the functional chain of dependencies that binds a provided service to required ones. On this example, it is easy to explain the functional dependencies that binds the getLocation service to the getSignal services, through the assemblies and the delegations.

As the extra-functional properties are intrinsic features of a service, the functional dependency implies an extra-functional dependency. On our example, consider the 
GPS component: it is obvious that the getLocation service will be completed after the getSignal services. This relationship can be explained as a constraint that binds $\theta_{\mathbf{C}}$ and the whole of $\theta_{\mathrm{s}}$ :

$$
\theta_{\mathbf{C}} \geq \max \left(\theta_{\mathrm{S}}\right) .
$$

A more detailed analysis of the behavior of the GPS (Fig. 1), and particularly of the functional chain of dependencies that binds the getLocation service to the getSignal service, implies that:

$$
\begin{gathered}
\theta_{\mathbf{R}}=\theta_{\mathbf{S}}+2, \\
\theta_{\mathbf{D}}=\max \left(\theta_{\mathbf{R}}\right)+3, \\
\theta_{\mathbf{C}}=\theta_{\mathbf{D}}+\mathbf{n b r} * \log (\mathbf{n b r}) .
\end{gathered}
$$

where :

- $\theta_{\mathrm{S}}$ is the response-time of the getSignal service, i.e. the time spent by a receiver to acquire a complete signal from a satellite,

- $\quad \theta_{\mathbf{R}}$ is the response-time of the getInfo service, i.e. the reception time of a signal $\left(\theta_{\mathrm{S}}\right)$ more the time spent to demultiplex the signal $(2 \mathrm{~ms})$,

- $\theta_{\mathbf{D}}$ is the response-time of the getData service, i.e. the maximum of the $\theta_{\mathbf{R}}$, more the decoding time of demultiplexed signals $(3 \mathrm{~ms})$.

- $\quad$ nbr is the number of active receivers (i.e. the number of powered receivers that get a signal). This number has an impact on the response-time of the getLocation service $\left(\theta_{\mathbf{C}}\right)$ because of the interpolation algorithm used.

This example clearly illustrates how the functional dependency of services can be translated into an equivalent extra-functional chain of dependencies.

\subsection{The QoSCL metamodel}

In the previous section, we have introduced the concepts of quality, level of quality and extra-functional dependency. We propose to extend the UML2.0 metamodel with these concepts. This specific metamodel, called QoS Constraint Language (QoSCL), allows a designer to specify:

- the intrinsic qualities of a service,

- $\quad$ the required or provided quality levels of a service

- the extra-functional dependency of a provided service's quality on a set of qualities defined on required services.

The Fig. 2 below shown the QoSCL extension of the UML2.0 metamodel. We have defined four new classes:

- ComponentQoSCL: extends Component. In addition of its functional provided and required interfaces, it has specific provided and required ContractTypes.

- ContractType: is a specialized Interface, which all elements of its ownedOperation attribute are typed as Dimension. Moreover, a provided (resp. required) ContractType is associated to only one Operation that belongs to a provided (resp. required) Interface. 
- Contract: is a class encapsulated by a component that implements a ContractType. The implementation of the Dimensions depends on each component, and each contract has its own renegotiation behaviour. This behaviour can be specified with state charts, activity diagrams, etc...

- Dimension: extends Operation. It specifies the means of measurement of the effective quality level of an extra-functional property and, by assimilation, the property itself. The semantic of its three constraints is:

- body: the set of possible values for this extra-functional property;

- pre: the required quality level, which must be compliant with the body constraint;

- post: the provided quality level, which must be compliant with the two previous.

A Dimension has optionally ContractTypes as parameters that represents its extra-functional dependency with other qualities.

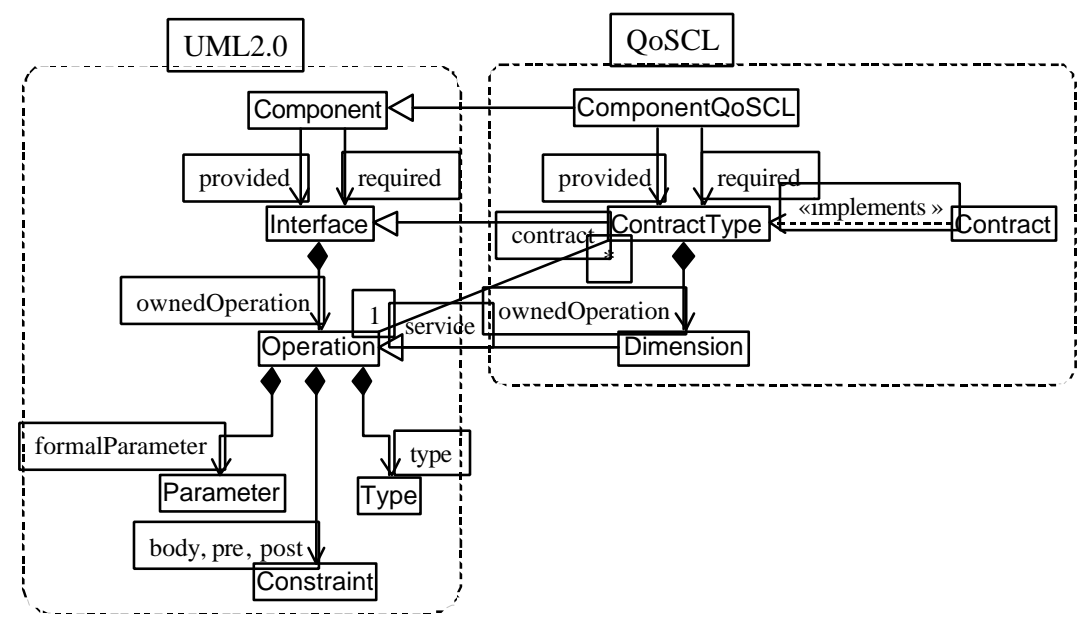

Fig. 2. The QoSCL metamodel

The Fig. 3 below shown the QoSCL model of the GPS component. The getLocation and getSignal services are connected their respective ContractTypes. The thetaC Dimension depends on the getSignal ContractType (attribute parameter). The thetaS Dimension has three constraints:

- $\quad$ its body specifies that its value must be positive;

- $\quad$ its post specifies that the getSignal service provided by the Satellite component is completed in more than $15 \mathrm{~ms}$ and less than $30 \mathrm{~ms}$;

- its pre specifies that the GPS component requires this service with a response time less than $15.5 \mathrm{~ms}$.

The thetaC post-condition ensures that the response time of the getLocation service is less than $24 \mathrm{~ms}$. This service depends on the getSignal service, and, as we have underline yet, this dependency implies an extra-functional dependency between their respective extra-functional properties. That is the reason why the qualitative level 
requirement on thetaC (i.e. its post-condition) implies a requirement on thetaS (i.e. its pre-condition). This mechanism will be more detailed in the following chapter of this paper.

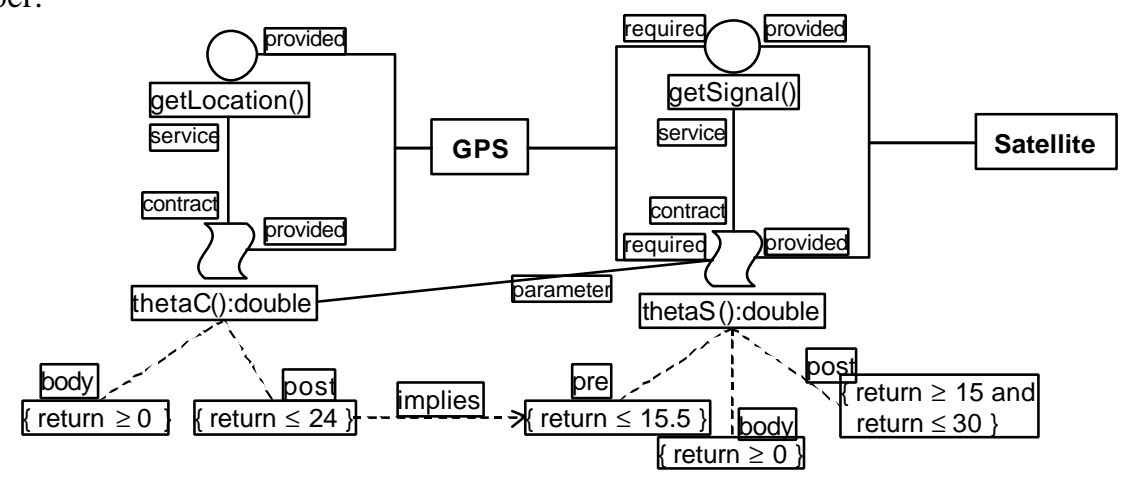

Fig. 3. The QoSCL model of the GPS

The QoSCL model is a platform independent component model, specifying abstract QoS properties and behaviors (ContractType and Dimension). It can be used to implement a concrete atefact, including monitors for the extra-functional properties that implement the specified behavior (Contract). Such implementations have already done: for instance, in the QCCS European project [10], an aspect weaver has been implemented in order to weave QoS properties into a functional platform independent model. A model transformation [11] translates this model into a .Net platform dependant model that implements a set of monitors and renegotiable contracts.

\section{Predict the QoS levels}

At this point, we have a QoS specification language, QoSCL, that allows to refine a UML2.0 component model with QoS properties. This language can support the implementation of the QoS concepts such as monitors and contracts, thanks to aspect weaving techniques followed by a model transformation for example. However, at design time, it is also possible to implement a tool that predicts the QoS levels according to the QoSCL specifications.

\subsection{QoS levels prediction features}

In the beginning of the QoS concepts section (\$2.1), we have said that a QoS property is a valuable quantity. It supports a set of constraints and it is bonded to others QoS properties via the extra-functional dependencies $(\mathbf{1 , 2 , 3 , 4})$. So the first important point to underline is that QoSCL allows to declare a set of QoS properties and their relations. 
The second important point is the form of the declared relations: constraints (required quality levels), mathematical equations (1-4) or empirical rules. These two last forms are shown on the two figures below (Fig. 4, Fig. 5):

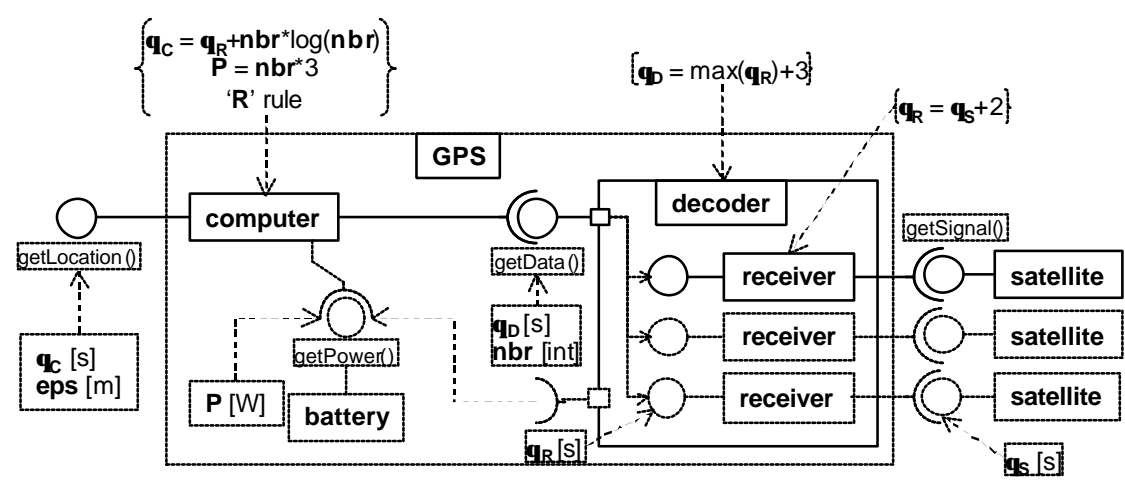

Fig. 4. GPS component model refined with its declared QoS properties and relations

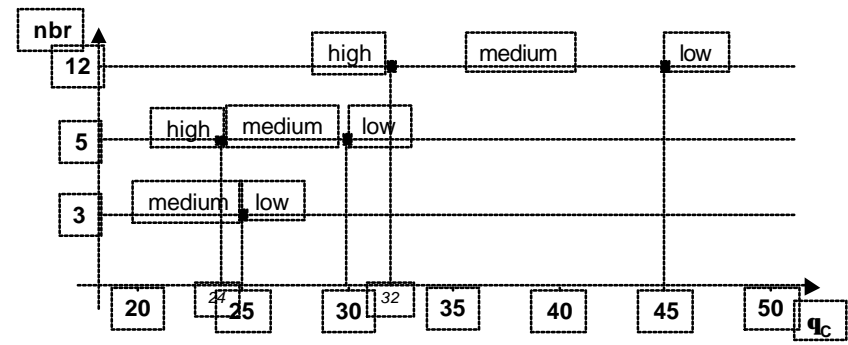

Fig. 5. The ' $R$ ' rule that binds three $Q o S$ properties (eps, nbr and $\theta_{C}$ )

where $\mathbf{P}$ is the reactive power consumed by the computer and decoder components. It is an extra-functional property of the getPower service.

Our GPS model has twelve receivers (only three shown in the previous figures). However, all the receivers are not powered, in order to manage the power consumption, and only a subset of them receive a clear satellite signal. This subset depends on the environment: for example, this set is empty in a tunnel that blocks the signals transmission. The nbr property is equal to the number of powered receivers that have a clear signal.

The third important point of the QoS levels prediction is the nature of the information that we want to handle and to give at the designer. The QoS properties belong to valued sets, and that is this high-level information will be handled. Indeed, for engineers, a symbolic relation that binds a set of variables is not intuitive: it not allows an immediate knowledge. The only form that a human actor like a designer can easily understand is a valued set, i.e. either a finite set of values or a numerical intervalFor instance, on the Fig. 3 diagram, the designer knows that the response time $\theta_{\mathbf{S}}$ of the getSignal service belongs to the real interval $[15 ; 15.5]$. An empty set would have 
implied an error, i.e. that the service would not be provided with a quality level compliant with the set of declared constraints and dependencies.

At last, we want to allow the designer to study the influence of a specific quality level requirement on any QoS property on the others. In other words, the designer wants to study the propagation of an information through a network of relations. This mechanism is more complex than an evaluation of a set of functions.

For instance, we present below two information propagations through the network of relations shown in Fig. 4:

$$
\begin{gathered}
\theta_{\mathbf{S}} \in[15 ; 30], \mathbf{n b r} \in\{3,5,12\} \Rightarrow \\
\theta_{\mathbf{R}} \in[17 ; 32], \theta_{\mathbf{D}} \in[20 ; 35], \theta_{\mathbf{C}} \in[21.43 ; 47.95] . \\
\text { eps }=\text { high, } \mathbf{P} \leq 15, \mathbf{n b r} \in\{3,5,12\} \Rightarrow \\
\theta_{\mathbf{C}} \in[23.49 ; 24], \theta_{\mathbf{D}} \in[20 ; 20.5], \theta_{\mathbf{R}} \in[17 ; 17.5], \theta_{\mathbf{S}} \in[17 ; 17.5] .
\end{gathered}
$$

As a consequence of this last point, the propagation of information through the network of relations implies the conformance of the pre, post and body conditions of each extra-functional property defined on an assembly.

\subsection{Implementation details}

To resume our problem, QoSCL allows a designer to declare a set of extra-functional properties, bonded between them into a network of relations. Theses relations are either numerical constraints (the quality levels), mathematical (non-linear) functions $(\mathbf{2 , 3 , 4 )}$ or empirical rules (Fig. 5). The designer wants to select any extra-functional properties and to observe the propagation of a qualitative level requirement on the others properties through the network. The result that he obtains must be under the same form as its requirement, i.e. a valued set.

Finally, we want the following features:

1. a declarative technology;

2. a network of relations that binds a set of variables;

3. relations as constraints, mathematical non-linear functions or rules;

4. information as valued sets (finite sets or numerical intervals).

The only technology that covers the whole of these four points is the Constraint Logic Programming (CLP) [12] including a real interval arithmetic solver, denoted CLP(R) [13]. The tools that implement this particular technology are few: PrologIV [14] and Eclipse [15]. There exist also interval arithmetic solvers only, such as RealPaver [16]. These software handle intervals, which are reduced by considering the constraints. The result is the most reduced interval including the solution, if there exists. Here is an example [ref $p 4]$ :

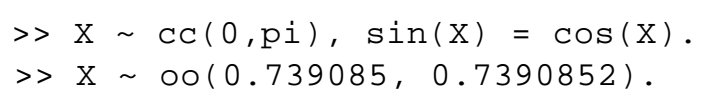


It is important to note that many CLP software declare to integrate a CLP(R) solver, like SICStus-Prolog [17] for example. However, their solvers are not based on the interval arithmetic, but on a symbolic computation, which does not handle interval and fails to compute non-linear constraints:

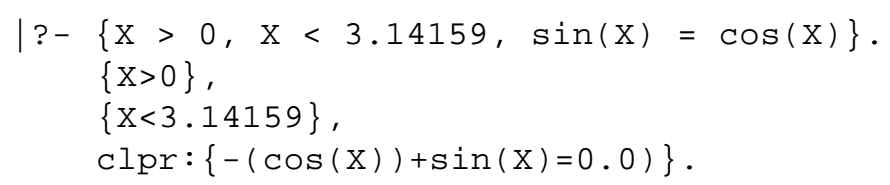

The QoS specifications are written in QoSCL. Although this language has all the features of a CLP compliant language, it was not originally dedicated to this specific use. Implementing a dedicated CLP solver engine, with its interval arithmetic solver, is not an easy task. Because of the common features, it is more judicious to transform QoSCL into an existing CLP language, using a MOF compliant model transformation such as MTL [11]. The result of a transformation of the GPS comp onent-based model with QoSCL into a PrologIV program for example is:

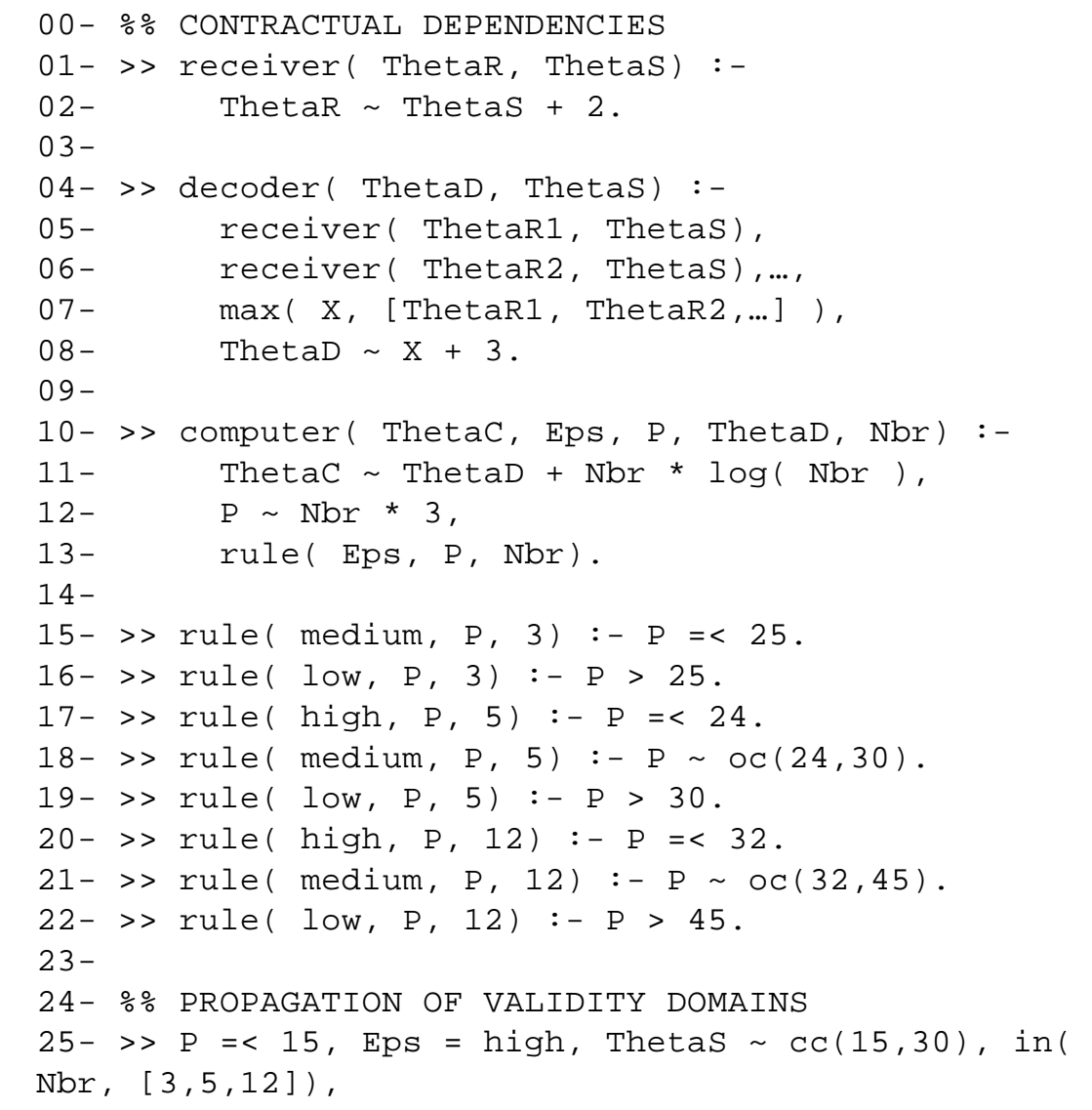


27- computer( ThetaC, Eps, P, ThetaD, Nbr).

The three first predicates (receiver/2, decoder/2 and computer/5) reify the dependencies of the extra-functional properties defined on each component. The rule/3 predicate is used by the computer $/ 5$ predicate in order to bind the Eps, $P$ and $N b$ Fveralizbleke 5 to 27 is a request (6), which is made up of two parts:

1. line 25: the ThetaS (resp. Nbr, P, Eps) variable belongs to the [15;30] (resp. $\{3,5,12\},[0 ; 15],\{$ high $\}$ ). These numerical constraints are either induced by the specifications and the environment, or by a designer who wants to know the global extra-functional impact of a specific stress applied on one or more properties.

2. line 26-27: represents the connection between the components, extracted from the component-based model. The extra-functional properties can be shared at this level between two components, like the ThetaD variable is shared by the decoder and the computer. It is also a request that the designer can answer. The answer computed by the inference engine is:

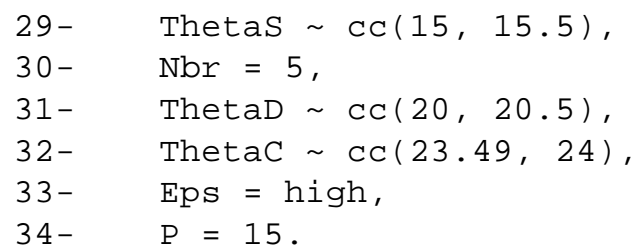

The result is a piece of PrologIV code, which is not very easy to understand. The semantic of this result and the chaining of the rules by the inference engine are explained in the following section.

\subsection{Inference and result}

In a CLP software, it is important to underline the fact that the use of numerical predicates $(+/ 3,-/ 3, \ldots)$ on a variable implies that this variable belongs to the real $\mathrm{R}$ or the integer $\mathrm{N}$ sets by construction. However, this kind of information induces few new knowledge by propagation and reduction of the intervals. That is the reason why, in line 25, we stress some properties in order to reduce their validity domains. These constraints are either provided by the specifications of the device $\theta_{\mathbf{S}} \in[15 ; 30]$, $\mathbf{n b r} \in\{3 ; 5 ; 12\})$ or by designer's requirements $(\mathbf{P} \leq 15, \mathbf{e p s}=$ high $)$.

The computation of the current location has a power cost $\mathbf{P}$, which depends on the number of active receivers $\mathbf{n b r}$ according the simple formula:

$$
\mathbf{P}=\mathbf{n b r} * 3 .
$$

According to (7) and the requirement that the power must be less or equal than 15 , we obtain that the number of active receivers must be equal to 3 or 5 : 


$$
\text { nbr } \in\{3 ; 5\} .
$$

The lines 15 to 22 express the rule that binds the precision eps, the response time $\theta_{\mathbf{C}}$ and the power $\mathbf{P}$ of the getLocation service (Fig. 5). According to this rule, the constraints (7) and (8), and the fact that eps is required to be high, it is obvious that:

$$
\begin{aligned}
& \mathbf{n b r}=3, \\
& \mathbf{P}=15 .
\end{aligned}
$$

The relationship that binds the response time $\theta_{\mathbf{C}}$ to the response time $\theta_{\mathbf{D}}$ and the number of active receivers $\mathbf{n b r}$ is:

$$
\theta_{\mathbf{C}}=\theta_{\mathbf{D}}+\mathbf{n b r} * \log (\mathbf{n b r}) .
$$

The second term $(\mathbf{n b r} * \log (\mathbf{n b r}))$ is the time spend by the computer to interpolates the position from the data. Since the validity domain of $\theta_{\mathbf{D}}$ and the value of nbr are known, we have:

$$
\theta_{\mathrm{C}} \in[23.49 ; 24] \text {. }
$$

This interval can be propagated again through the response time chain of dependencies (Fig. 4), and finally we obtain the result on $\theta_{\mathbf{D}}$ and $\theta_{\mathbf{S}}$ :

$$
\begin{aligned}
& \theta_{\mathbf{D}} \in[20 ; 20.5], \\
& \theta_{\mathbf{S}} \in[15 ; 15.5] .
\end{aligned}
$$

That is the result obtain by the CLP software (1. 29-34).

\section{Related works}

QoS and extra-functional properties are not a new concept in the component-based software engineering [1][5]. Many authors have developed languages dedicated to extra-functional specifications: SMIL [18], TINA-ODL [19], QIDL [20], QDL [21] and so on. An interesting analysis of these languages and their common features is done by Aagedal [22]. He concludes that the QoS Modeling Language (QML) [9] is the most general language for QoS specification. Indeed, the concepts defined in QML are representative of specifications languages dedicated to the extra-functional aspects. These concepts have been integrated into QoSCL.

QML has three main abstraction mechanisms for the QoS specification: contract type, contract and profile. A contract type represents a specific QoS aspect, such as reliability for example. It defines valuable dimensions, used to characterize a particular QoS aspect. A contract is an instance of a contract type and represents a particular QoS specification. Finally, QoS profiles as sociate contracts with interfaces.

With QML, it is possible to write a contract which specifies that an operation must be completed with a delay less than 30 seconds: 


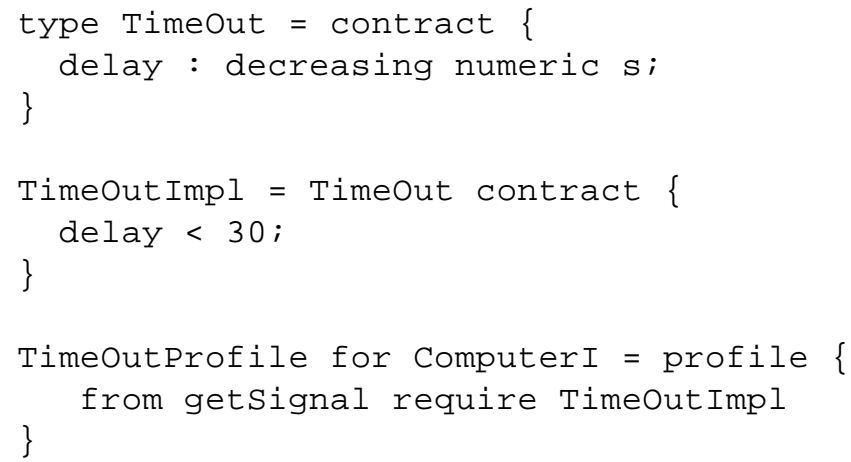

In spite of its generality, QML, as the other languages mentioned above, does not have explicit dependencies between extra-functional properties. The properties are considered as independent quantities, evaluated at run time by monitors. But that does not match reality: we have shown that extra-functional properties have dependencies in the same way as a provided service depends on a set of required services. QoSCL makes these dependencies explicit.

The QoS specifications can be used either to implement contracts evaluation and renegotiation at run time, or to evaluate a priori the global quality of the assembly at design time.

G. Blair has proposed a specific architecture in order $\mathbf{b}$ manage component composition, based on a reflective component model [23][24]. In fact, the component reflection is dedicated to access at properties value and structural information of the composite. A set of rules, called StyleRule, manages the adaptation of the composite to its environment. In fact, these rules can be considered as model transformations used to reconfigure the composite: properties values, connections between components and graph actions.

In the various models shown, the extra-functional properties are not explicitly defined, neither their dependencies a fortiori. Consequently, it is not possible to predict at design time the global quality of the assembly.

Moreover, according to us, the use of rules in order to dynamically configure an assembly at run time is dangerous. Indeed, the authors of [25], which have implemented a declarative approach for adaptative components, underline the limit of such approach: the set of rules which governs the adaptation must respect completeness and uniqueness.

Completeness guarantees that for every possible situation there exits at least one adaptation action, and uniqueness ensures that this action is unique. The authors indicates that the two following properties can be enforced by use of the CLP. However, not only these two properties are not compatible with non-linear constraints [25], but also the extra-functional dependencies can be non-linear (see formula \#4).

That is the reasons why we have chosen to implement the contracts evaluation and renegotiation in imperative language with a weaving aspect technology. Like Genßler and Zeidler [26], or in the Itacio tool [27], the use of CLP is kept till the design time, to check the validity of an assembly according to a set of rules (consistency rules, 
contractual rules, etc..). However, none of them exhibits a component model with explicit extra-functional dependencies.

At last, researchers of the Software Engineering Institute at Carnegie Mellon University (USA) have underlined the importance to integrate more analysis technology in components-based software [28]. We think that this integration must be considered at the highest level: the component model. QoSCL (specification) and its dedicated tools (validation and renegotiation) presented in this paper are a contribution in this sense.

\section{Conclusion and future work}

In mission-critical component based systems, it is particularly important to be able to explicitly relate the QoS contracts attached to provided interfaces of components with the QoS contracts obtained from their required interfaces. In this paper we have introduced a language called QoSCL (defined as an add-on to the UML2.0 comp onent model) to let the designer explicitly describe and manipulate these higher level contracts and their dependencies.

An important feature of QoSCL is that this language distinguishes four degrees of conformance for component-based diagram refined with extra-functional properties:

- $\quad 1^{\text {st }}$ degree: the functional conformance. That is the well-known conformance between provided and required typed interfaces.

- $2^{\text {nd }}$ degree: because the QoSCL ContractTypes are interfaces which operations are Dimensions, the extra-functional conformance is very close of the previous degree, applied to the ContractTypes of a component.

- $\quad 3^{\text {rd }}$ degree: each extra-functional property is a valuable quantity, constrained by body, pre and post conditions. At the assembly level, the conformance of these three constraints must be checked.

- $\quad 4^{\text {th }}$ degree: the quality levels of the whole of the extra-functional properties must be compliant with the networks of (non-linear) relations.

The other main feature of QoSCL contracts is that they can be exploited for:

- validation of individual components, by automatically weaving contract monitoring code into the components for example;

- validation of a component assembly, including getting end-to-end QoS information inferred from individual component contracts, by automatic translation into a CLP(R) language.

Both validation activities builds on the model transformation framework developed at INRIA (cf. http://modelware.inria.fr). Preliminary implementations of these ideas have been prototyped in the context of the QCCS project (cf. http://www.qces.org) for the weaving of contract monitoring code into components part, and on the Artist project (http://www.systemes-critiques.org/ARTIST) for the validation of a component assembly part. Both parts still need to be better integrated with UML2.0 modelling environments, which is work in progress. 


\section{Acknowledgments}

This work has been partly founded by the ARTIST IST project. The authors thank Jean-Philippe Thibault for the discussions.

\section{References}

1. "Component software, beyond object-oriented programming", $2^{\text {nd }}$ ed., by C. Szyperski. Addison-Wesley, 2002

2. "CORBA Components, v3.0", adopted specification of the OMG, June 2002.

3. "EJB 2.1 Specification Final Release", Sun, 2002.

4. "Applied Microsoft .Net framework programming" by J. Richter. Microsoft Press, January 23, 2002.

5. "Synchronization in concurrent object-oriented languages:expressive power, genericity and inheritance" by C. McHale. Doctoral dissertation, Trinity College, Dept. of computer science, Dublin, 1994.

6. "Making components contract aware" by A. Beugnard, J.M. Jézéquel, N. Plouzeau and D Watkins in Computer, pp. 38-45, IEEE Computer Society, July 1999.

7. "UML Superstructure 2.0", OMG, August 2003.

8. "Constraint logic programming" by J. Jaffar and Lassez J.L. in proceedings of $14^{\text {th }}$ ACM Symposium on principles of programming languages (POPL'87), pp-111-119, ACM, 1987

9. "QoS specification in distributed object systems" by S. Frolund and J. Koistinen in Distributed Systems Engineering, vol. 5, July 1998, The British Computer Society

10. http://www.qces.org, Quality Control of Component-based Software (QCCS) European project home page.

11. "Reflective model driven engineering" by J. Bézivin, N. Farcet, J.-M. Jézéquel, B. Langlois, and D. Pollet in Proceedings of UML 2003, San Francisco, volume 2863 of LNCS, pages 175-189. Springer, October 2003.

12. "Logical arithmetic" by J.G. Cleary in Future computing systems 2, n², pp-125-149, 1987

13. "Applying interval arithmetic to real, integer and Boolean constraints" by F. Benhamou and W.J. Older, in Journal of logic programming 32, n 1, pp-1-24, 1997

14. "PrologIV: reference manual and user's guide", PrologIA, Tech. Rep., 1994

15. "Hybrid Problem Solving in ECLiPSe", by F. Ajili and M. Wallace, §6 in "Constraint and integer programming: toward a unified methodology”, pp. 169-201. Michela Milano. Kluwer Academic Publishers. October 2003.

16. "An interval component for continuous constraints". Journal of Computational and Applied Mathematics, 2003.

17. http://www.sics.se/sicstus/

18. "Synchronized Multimedia Integration Language 2.0 specification" by W3C: http://www.w3.org/TR/smil20

19. “TINA object definition language manual”, TINA-C, report : TP_NM_002_2.2_96, 1996

20. "QoS, aspects of distributed programs" by C. Becker and K. Geiths, in proceedings of international workshop on aspect-oriented programming at ICSEE'98, Kyoto, Japan, 1998. 
21. "Integration of QoS in distributed objects systems" by J. Daniels, B. Traverson and S. Vignes, in proceedings of IFIP TC6 WG6.1 2nd international working conference on Distributed Applications and Interoperable Systems (DAIS'99), Helsinki, Finland, pp. 31-43, 1999.

22. "Quality of service support in development of distributed systems" by J.O. Aagedal. Ph.D thesis report, University of Oslo, Dept. Informatics, March 2001.

23. "v-QoS project home page": http://www.comp.lancs.ac.uk/computing/users/lb/v-qos.html

24. "A reflective and architecture aware component model for middleware composition management" by G.S. Blair, R. Silva Moreira and E.M. Carrapatose in $3^{\text {d }}$ international symposium on Distributed Objects and Application (DOA'01), September 2001, Roma (It).

25. " A declarative approach for designing and developing adaptative components" by $P$.

Boinot, R. Marlet, G. Muller and C. Consel, in $15^{\text {th }}$ international conference on Automated Software Engineering (ASE'01), pp-111-119, September 2000, Grenoble (Fr).

26. "Rule-driven component composition for embedded systems" by. T. Genssler and C. Zeidler in $4^{\text {th }}$ ICSE workshop on component-based software engineering: component certification and system prediction, ICSE'01, Toronto $(\mathrm{Ca})$.

27. "Itacio: a component model for verifying software at construction time" by A. Cernuda del Rio, J.E. Labra Gayo and J.M. Cueva Lovelle in international workshop on componentbased engineering, ICSE'00, Mimerick (Ir).

28. "Packaging and deploying predictable assembly" by S.A. Hissam, G.A. Moreno, J. Stafford and K.C. Wallnau, in the proceedings of IFIP/ACM working conference on component deployment (CD2002), , pp. 108-124, Berlin, Germany, June 2002. 